\title{
Tumor dissemination through surgical tracts in diffuse intrinsic pontine glioma
}

\author{
Maria-Jesus Lobon-Iglesias, MD,1,2 Vicente Santa-Maria Lopez, MD, 3,4 \\ Patricia Puerta Roldan, MD, ${ }^{5}$ Santiago Candela-Cantó, MD, ${ }^{5}$ Monica Ramos-Albiac, MD, ${ }^{6}$ \\ Marta Gomez-Chiari, MD, ${ }^{7}$ Stephanie Puget, MD, PhD, ${ }^{8}$ Stephanie Bolle, MD, ${ }^{9}$ \\ Liliana Goumnerova, MD, ${ }^{10}$ Mark W. Kieran, MD, PhD, ${ }^{11}$ Ofelia Cruz, MD, PhD, ${ }^{3,4}$ \\ Jacques Grill, MD, PhD, ${ }^{1,2}$ and Andres Morales La Madrid, MD,4
}

\begin{abstract}
'Department of Pediatric and Adolescent Oncology and ${ }^{~}$ Department of Radiation Therapy, Gustave Roussy and University Paris-Saclay, Villejuif; ${ }^{2}$ Team "Target Identification and Innovative Anticancer Therapies in Pediatric Cancers," Centre National de la Recherche Scientifique Unité Mixte de Recherche 8203, Villejuif; ${ }^{8}$ Department of Pediatric Neurosurgery, Necker Sick Children's Hospital and University Paris-Descartes, Paris, France; ${ }^{3}$ Department of Pediatric Hematology and Oncology, ${ }^{4}$ Pediatric Neuro-Oncology, Department of Pediatric Hematology and Oncology, ${ }^{5}$ Department of Pediatric Neurosurgery, and 'Department of Radiology, Hospital Sant Joan de Déu; ${ }^{6}$ Department of Radiation Oncology, Hospital Vall d'Hebron, Barcelona, Spain; ${ }^{10}$ Department of Neurosurgery, Boston Children's Hospital; and ${ }^{11}$ The Pediatric Brain Tumor Program, Department of Pediatric Oncology, Dana-Farber Boston Children's Cancer and Blood Disorders Center, Boston, Massachusetts
\end{abstract}

OBJECTIVE Diffuse intrinsic pontine glioma (DIPG) is a highly aggressive and lethal brainstem tumor in children. In the 1980s, routine biopsy at presentation was abandoned since it was claimed "unnecessary" for diagnosis. In the last decade, however, several groups have reincorporated this procedure as standard of care or in the context of clinical trials. Expert neurosurgical teams report no mortality and acceptable morbidity, and no relevant complications have been previously described. The aim of this study was to review needle tract dissemination as a potential complication in DIPG.

METHODS The authors retrospectively analyzed the incidence of dissemination through surgical tracts in DIPG patients who underwent biopsy procedures at diagnosis in 3 dedicated centers. Clinical records and images as well as radiation dosimetry from diagnosis to relapse were reviewed.

RESULTS Four patients ( 2 boys and 2 girls, age range $6-12$ years) had surgical tract dissemination: in 3 cases in the needle tract and in 1 case in the Ommaya catheter tract. The median time from biopsy to identification of dissemination was 5 months (range 4-6 months). The median overall survival was 11 months (range 7-12 months). Disseminated lesions were in the marginal radiotherapy field $(n=2)$, out of the field $(n=1)$, and in the radiotherapy field $(n=1)$.

CONCLUSIONS Although surgical tract dissemination in DIPG is a rare complication (associated with $2.4 \%$ of procedures in this study), it should be mentioned to patients and family when procedures involving a surgical tract are proposed. The inclusion of the needle tract in the radiotherapy field may have only limited benefit. Future studies are warranted to explore the benefit of larger radiotherapy fields in patients with DIPG.

https://thejns.org/doi/abs/10.3171/2018.6.PEDS17658

KEYWORDS DIPG; dissemination; biopsy; oncology

ABBREVIATIONS CED = convection-enhanced delivery; DFCI = Dana-Farber Cancer Institute; DIPG = diffuse intrinsic pontine glioma; GR = Gustave Roussy; HGG = high-grade glioma; PTV = planning target volume; RT = radiotherapy; SJD = Sant Joan de Déu Hospital.

SUBMITTED November 21, 2017. ACCEPTED June 12, 2018.

INCLUDE WHEN CITING Published online September 7, 2018; DOI: 10.3171/2018.6.PEDS17658. 
$\mathrm{D}$ IFFUSE intrinsic pontine glioma (DIPG) accounts for the vast majority of brainstem tumors in children. Upfront focal irradiation is the only effective palliative therapy aiming at delaying tumor progression..$^{13}$ In most centers, diagnosis is performed based upon classic clinical presentation and MRI radiological criteria. ${ }^{13,18}$ Thirty years ago, surgical biopsy of these lesions was abandoned in most suspected cases, supported by the hypothesis that the information obtained by morphological examination had no impact on the diagnosis, prognosis, or management and was associated with significant morbidity. ${ }^{12}$ In the last decade, advances in neurosurgical technique and molecular biology have permitted molecular characterization of DIPG, defining discrete subgroups with different molecular alterations, and several centers have started performing tumor biopsies with good diagnostic yield and sufficient tumoral tissue for molecular diagnosis and research. 2,4,5,11,14-16,19 Thus, DIPG is no longer seen as a single entity, but as a heterogeneous group of tumors that develop in the ventral pons and harbor both specific genetic (i.e., TP53, PDGFRA, ACVRI, and FGFRI) and epigenetic alterations (i.e., K27M H3.3 and H3.1 variants). This molecular variability may explain different tumor behavior and response to radiation therapy. However, these subgroups still share the same dismal prognosis. The information obtained from upfront biopsies of DIPG has opened an avenue for intensive preclinical and clinical research that will hopefully translate into more effective therapies within the next few years. Under strict guidelines at centers of excellence, the diagnostic surgical biopsy has acceptable morbidity, with only transient neurological deterioration in a small number of patients, persistent neurological deficits in less than $5 \%$ of patients, and no mortality reported. Recently, however, out of 23 procedures performed in French referral centers, 2 cases of tumor dissemination associated with the stereotactic needle tract biopsy were identified, ${ }^{10}$ prompting us to examine our series of DIPG biopsies to better define its frequency and to explore potential risk factors and preventive strategies. DIPGs may develop cystic degeneration that may prompt treatment teams to place an Ommaya catheter, and there is growing interest in exploring convection-enhanced delivery (CED) for this patient population. These interventions, which involve surgical tracts, may thus also facilitate tumor cell dissemination.

We report 3 cases of tumor dissemination within biopsy needle tracts and 1 case of tumor dissemination within an Ommaya catheter tract.

\section{Methods}

A multicenter retrospective review of all DIPG surgical procedures was performed in the databases from 2 centers: Gustave Roussy (GR) (biopsies performed at Necker Hospital, Paris, France) and Sant Joan de Déu Hospital (SJD) (Barcelona, Spain). Patients younger than 18 years and fulfilling the following criteria were eligible: 1) clinical and radiological signs of a typical DIPG at primary diagnosis, 2) tumor biopsy performed at diagnosis, 3) other surgical procedures performed, and 4) well-documented postsurgical imaging identifying the surgical tract. We reviewed imaging evaluations from diagnosis to progression and death. Demographics, progression-free survival, and outcome were also analyzed. Of note, we collected radiotherapy (RT) treatment plans to analyze whether the needle tract dissemination lesions were included in the upfront RT fields. We considered disseminated lesions to be in the RT field if $95 \%$ of them were situated within the planning target volume (PTV), marginal if $20 \%-95 \%$ were in the PTV, and out of the field if less than $20 \%$ of disseminated lesions were in the PTV. Additionally, we describe a third case with biopsy needle dissemination from the Dana-Farber Cancer Institute (DFCI) (Boston, Massachusetts).

\section{Results}

\section{Study Population}

One hundred twenty-five patients diagnosed at GR and SJD from January 2005 through December 2016 were included. We identified 3 patients (2.4\%) who developed disease dissemination during their disease course-in the biopsy needle tract in 2 cases and in the Ommaya catheter tract in 1 case. A fourth case identified at DFCI was also included in this report. The patients' median age at diagnosis of DIPG was 7.76 years (range 6.1-11.9 years). The median time from biopsy to identification of the needle tract dissemination was 5 months (range 4-6 months). The case of tumor dissemination in the Ommaya catheter tract was identified 4 months after the surgical procedure.

Of note, we found that 8 of 25 metastatic relapses were localized at the cerebellum but only 2 of them were related to the surgical tract (GR series, data not shown).

\section{Surgical Biopsies and Molecular Profile}

The stereotactic biopsy was performed under general anesthesia using either the Leksell frame (in Paris) or the frameless stereotaxic protocol and head fixation in a 3-point Mayfield clamp (in Barcelona). Intraoperative image guidance and the surgical plan were obtained using the Brainlab VarioGuide system. This technique was similar in those patients who did not experience needle tract dissemination. There were no immediate postprocedure complications reported.

In terms of histological review, the biopsy samples in the 4 cases of tumor dissemination associated with a surgical tract were concordant with anaplastic oligodendroglioma (1 case) and anaplastic astrocytomas (3 cases) (following the 2007 WHO Classification of Tumors of the Central Nervous System).

Molecular profiling showed all 4 cases to be positive for the $\mathrm{K} 27 \mathrm{M}$ mutations in the histone $\mathrm{H} 3$ gene H3F3A. Mutations in $A C V R I$ and $P I 3 K$ were also identified in case 4.

\section{Therapy Received, Evolution, and Outcome}

Three of the 4 patients were treated with upfront RT and one (case 4) received immunotherapy (Newcastle disease [NCD] virus and dendritic cell vaccination) prior to RT. Conventional RT was performed in all cases: fractionated focal irradiation to the tumor along with $1-$ to $2-\mathrm{cm}$ margins (54-60 Gy, 1.8- to 2-Gy fractions, given once daily for 5 days per week over a period of 6 weeks). It was not substantially different in those patients who did not develop tumor spread. Adjuvant therapy received is shown in Table 1. 
TABLE 1. Case summary: demographic characteristics, treatment, and outcomes

\begin{tabular}{|c|c|c|c|c|c|c|c|c|}
\hline $\begin{array}{l}\text { Case } \\
\text { No. }\end{array}$ & $\begin{array}{l}\text { Age (yrs), } \\
\text { Sex }\end{array}$ & Molecular Profile & $\begin{array}{l}\mathrm{RT} \text { (dose in Gyl } \\
\text { fractionation) }\end{array}$ & Adjuvant Therapy & $\begin{array}{l}\text { Time to Dissemination } \\
\text { in Tract (mos) }\end{array}$ & $\begin{array}{l}\text { TTP1 } \\
\text { (mos) }\end{array}$ & $\begin{array}{l}\text { Localization of } \\
\text { Disseminated Lesions }\end{array}$ & $\begin{array}{c}\text { OS } \\
\text { (mos) }\end{array}$ \\
\hline 1 & $8, F$ & H3.3K27M; EGFR+ & 54/normo & Erlotinib, BVZ & 6 & 6 & Marginal field & 7 \\
\hline 2 & $6, M$ & H3.3K27M; BRAF WT & 39/hypo & VPA, CEL, Thd, FA, VP16 & 6 & 6 & Marginal field & 11 \\
\hline 3 & $8, F$ & H3.3K27M; PDGFRA+ & 54/normo & NCDV, Nivolumab & 4 & 4 & Out of field & 11 \\
\hline 4 & $12, \mathrm{M}$ & H3.3K27M; EGFR+ & 54/normo & Erlotinib & $4 \dagger$ & 9 & In field & 12 \\
\hline
\end{tabular}

BVZ = bevacizumab; CEL = celecoxib; FA = fenofibric acid; hypo = hypofractionated; NCDV = Newcastle disease virus; normo = normofractionated; OS = overall survival; Thd = thalidomide; TTP1 = time to first progression; VPA = valproic acid; VP16 = etoposide.

${ }^{*}$ Regarding RT field.

† Time from Ommaya reservoir placement.

The patient in case 4 presented with dizziness and poor general condition 6 weeks after the end of RT. MRI showed pseudoprogression with compressive cyst formation. The patient's condition improved after cyst resection, but the same symptoms and cyst formation reappeared 1 month later. In order to drain the recurrent cyst an Ommaya reservoir was placed.

Four patients experienced tumor dissemination in the surgical tract at first progression: in the biopsy needle tract in 3 cases (case 1, Fig. 1; case 2, Fig. 2; and case 3, Fig. 3) and in the Ommaya catheter tract in 1 case (case 4, Fig. 4). For this cohort of patients, the median progression-free survival was 6 months (range 4-9 months) and the median overall survival was 10.4 months (range 7-12 months).

\section{RT Fields and Needle Tract}

Upfront irradiation fields were reviewed in order to evaluate the inclusion of the needle tract lesions in the planned fields. We found that in cases 1 and 2, the needle tract dissemination lesions were in the marginal RT field. In case 4, the disseminated lesions along the Ommaya tract were in the RT field. In case 3, the 3 needle tract dissemination lesions were completely out of the radiotherapy field. Radiotherapy dosimetry data for cases 1 and 2 are show in Figs. 1 and 2.

\section{Discussion}

DIPG is the most common malignant brainstem tumor in children. With current management, the mean overall survival reported in most series is around $9-11$ months, ${ }^{8}$ and the majority of patients succumb to their disease within 2 years after diagnosis. Focal irradiation is the most effective palliative therapy, producing tumor shrinkage and improvement of symptoms in most cases. However, this effect only lasts for a few months, and then tumor regrowth occurs and neurological symptoms reappear. ${ }^{13,18}$ Re-irradiation at tumor progression has gained popularity among most centers, but life expectancy at this point is poor. ${ }^{3}$ In the last 30 years, most centers have not included diagnostic tumor biopsy as the "standard of care" for DIPG since it was deemed potentially harmful for patients and, most importantly, unnecessary for confirming the diagnosis. Additionally and more importantly, the histological information obtained did not change therapeutic decisions or affect prognosis. ${ }^{6}$ As a result of the moratorium on upfront biopsy, the molecular landscape of DIPG remained unstudied and no advances in treatment were identified. With improvements in neurosurgical techniques and the development of more sophisticated molecular profiling technology capable of processing ${ }^{6,11}$ scant tumor material,
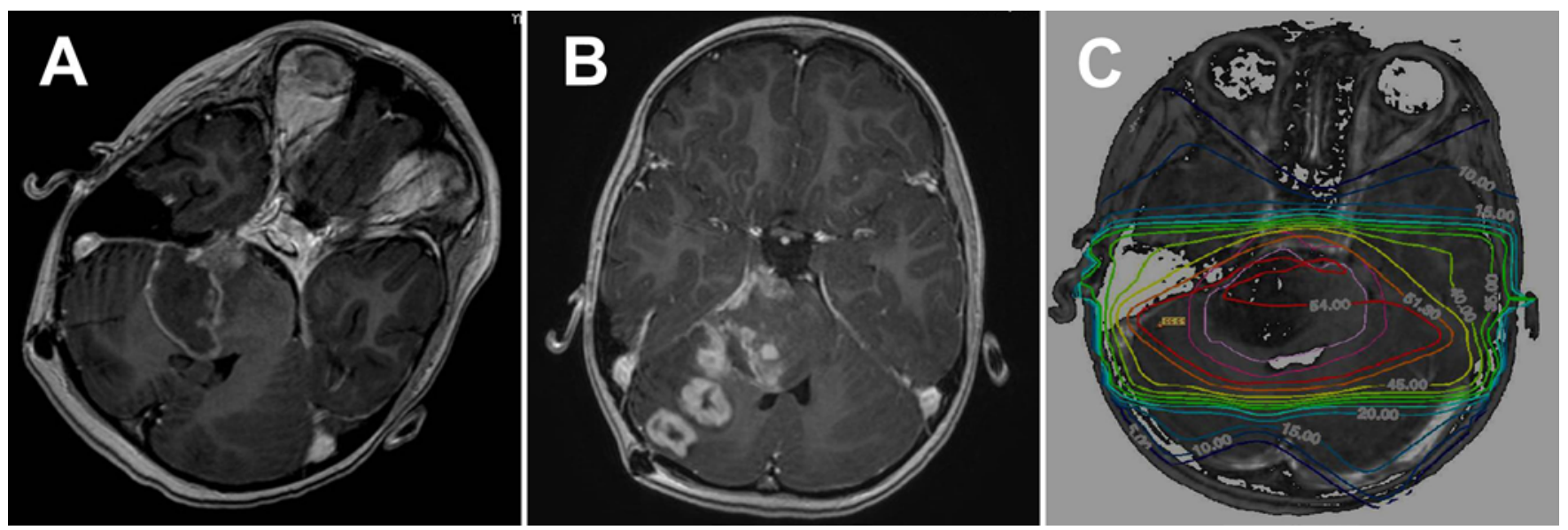

FIG. 1. Case 1. A and B: Axial Gd-enhanced T1-weighted MR images showing needle tract biopsy and pontine mass after biopsy (A) and pontine mass with needle tract dissemination at relapse (B). C: Image showing different radiotherapy fields with isodose lines. Figure is available in color online only. 

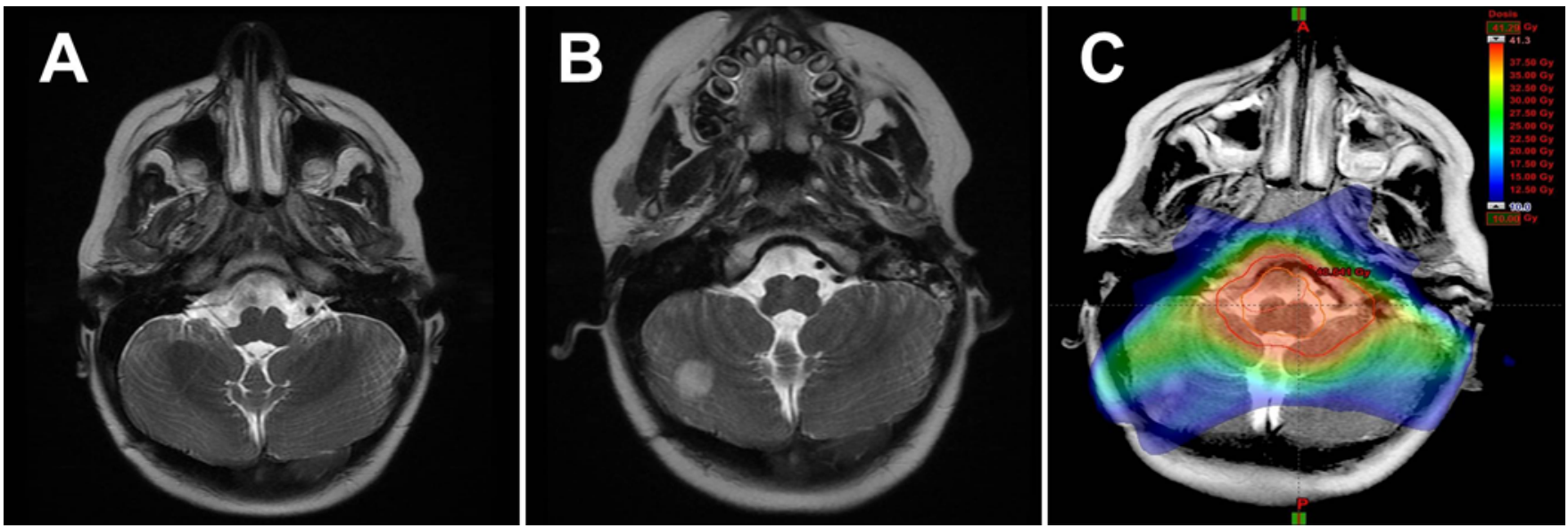

FIG. 2. Case 2. A and B: Axial T2-weighted MR images showing needle tract biopsy before (A) and after (B) DIPG relapse. C: Image showing needle tract dissemination with respect to the spatial relationship to the radiotherapy field. Figure is available in color online only.

several groups have safely performed minimally invasive diagnostic biopsies. Tumor molecular profiling has shown that DIPGs are associated with a group of unique molecular abnormalities when compared with other non-midline pediatric high-grade gliomas (HGGs) and adult gliomas, namely frequent $\mathrm{K} 27 \mathrm{M}$ mutations in one of the histone H3 genes. ${ }^{2,4,5,11,14-16,19}$ Added to this are different signaling pathway mutations $(A C V R 1, P I 3 K)$ or amplifications $(P D G F R)$ that suggest that DIPG represents a spectrum of tumors that develop in a narrow age range and in a specific area of the pons. This information will be paramount for the design of future clinical trials. ${ }^{9}$ However, the precious biological information obtained through surgical biopsy, necessary to refine therapeutic decisions or to include patients in clinical trials based on specific molecular alterations (NCT01182350 and NCT02233049, clinicaltrials. gov), may come with a cost. Most series published to date recognize that transient neurological deterioration may occur in a subset of patients, but more serious sequelae have been infrequently reported. Moreover, as the number of biopsies performed increases, it is likely that additional complications may be identified.
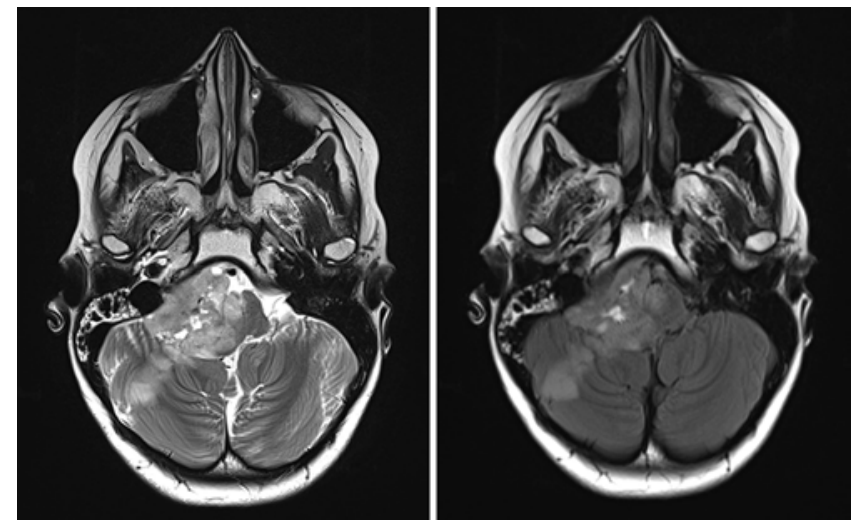

FIG. 3. Case 3. Axial gadolinium-enhanced T1-weighted (left) and axial T2-weighted (right) MR images showing biopsy needle tract dissemination.
Microscopic or macroscopic tumor dissemination in DIPG, initially thought to be very rare, is being recognized with increasing frequency as more imaging and postmortem examinations are performed, showing the potential of these tumor cells for migration and invasiveness. ${ }^{1}$ Nevertheless, tumor dissemination associated with the biopsy needle tract has been only recently reported. ${ }^{10}$ Prior to this, dissemination had only been associated with surgical biopsy in supratentorial $\mathrm{HGGs}^{7}$ but not in DIPG. However, given the invasive and diffuse nature of this tumor it is perhaps not surprising that this complication is being recognized more frequently. As the role of surgical biopsy and catheter placement for this patient population rises, it will be important to establish the true incidence of this complication. Newer delivery systems currently under clinical trials (e.g., CED; NCT03086616 and NCT01502917, clinicaltrials.gov) include placement of catheters, many of which will remain in place for extended periods to permit repeated therapy. Therefore, this information may be of relevance as this approach gains popularity.

Changes in RT fields, including the addition of the biopsy tract in the treatment plan, may "protect" from relapse/dissemination in the needle biopsy tract, potentially decreasing the likelihood of this complication, without significantly increasing the toxicity of upfront RT. Our review suggests that tumor dissemination in the needle biopsy tract is rare. Moreover, we found that progression-free survival and overall survival were not significantly altered by this complication. In addition, we found that distant relapses in the cerebellum were more frequently not associated with the surgical tract. Thus, the inclusion of the needle tract in the radiotherapy plan may only have limited benefit. Metastatic relapses have been described at higher frequency in DIPG ${ }^{17}$ but this has not involved changes in RT fields until now. Future studies are warranted to explore the benefit of larger RT fields in DIPG patients. Placement of intracystic catheters to treat tumoral cystic degeneration may also predispose to tumor dissemination. The hope is that in the near future, patients with DIPG may live longer thanks to novel delivery systems and more effective targeted thera- 

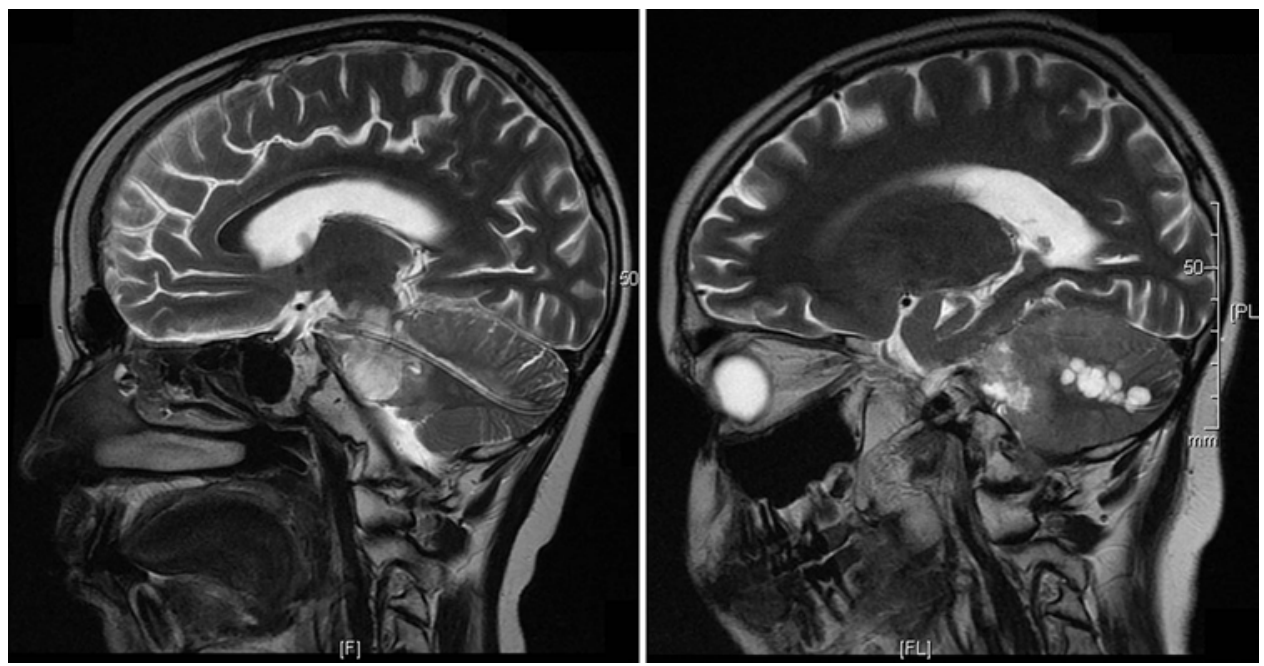

FIG. 4. Case 4. This patient experienced pseudoprogression with cyst formation needing cyst extirpation and intracyst Ommaya reservoir placement. Sagittal T2-weighted MR images show pontine lesion and reservoir Ommaya tract before relapse (left) and disseminated lesions in the intra-Ommaya reservoir tract at relapse (right).

pies. It is of paramount importance to standardize neurosurgical protocols with the goal of reducing the chance of contamination of tumor cells within the surgical tract. Collaborative efforts and dedicated task forces will be necessary to establish the safest neurosurgical technique and the appropriate radiation fields in order to minimize the risks associated with this procedure. Dissemination of the DIPG through the needle tract or along the catheter of an Ommaya reservoir is a worrisome but rare complication that is worth mentioning to patients and their family members when these procedures are to be performed. The evaluation of the impact of upfront stereotactic biopsies to tailor initial therapy is now ongoing in prospective trials; this evaluation will have to take into account all potential complications.

\section{Acknowledgments}

We thank all DIPG families that support our research initiatives, with special gratitude to the Alicia Pueyo Foundation.

\section{References}

1. Caretti V, Bugiani M, Freret M, Schellen P, Jansen M, van Vuurden D, et al: Subventricular spread of diffuse intrinsic pontine glioma. Acta Neuropathol 128:605-607, 2014

2. Castel D, Philippe C, Calmon R, Le Dret L, Truffaux N, Boddaert N, et al: Histone H3F3A and HIST1H3B K27M mutations define two subgroups of diffuse intrinsic pontine gliomas with different prognosis and phenotypes. Acta Neuropathol 130:815-827, 2015

3. Fontanilla HP, Pinnix CC, Ketonen LM, Woo SY, Vats TS, Rytting ME, et al: Palliative reirradiation for progressive diffuse intrinsic pontine glioma. Am J Clin Oncol 35:51-57, 2012

4. Fontebasso AM, Papillon-Cavanagh S, Schwartzentruber J, Nikbakht H, Gerges N, Fiset PO, et al: Recurrent somatic mutations in ACVR1 in pediatric midline high-grade astrocytoma. Nat Genet 46:462-466, 2014

5. Grill J, Puget S, Andreiuolo F, Philippe C, MacConaill L, Kieran MW: Critical oncogenic mutations in newly diagnosed pediatric diffuse intrinsic pontine glioma. Pediatr Blood Cancer 58:489-491, 2012

6. Kieran MW, Goumnerova LC, Prados M, Gupta N: Biopsy for diffuse intrinsic pontine glioma: a reappraisal. J Neurosurg Pediatr 18:390-391, 2016

7. Kim JE, Kim CY, Kim DG, Jung HW: Implantation metastasis along the stereotactic biopsy tract in anaplastic astrocytoma: a case report. J Neurooncol 61:215-218, 2003

8. Lobon-Iglesias MJ, Giraud G, Castel D, Philippe C, Debily MA, Briandet C, et al: Diffuse intrinsic pontine gliomas (DIPG) at recurrence: is there a window to test new therapies in some patients? J Neurooncol 137:111-118, 2018

9. Morales La Madrid A, Hashizume R, Kieran MW: Future clinical trials in DIPG: bringing epigenetics to the clinic. Front Oncol 5:148, 2015

10. Pierre-Aurélien B, Alexandru S, Federico DR, Justyna K, Carmine M, Didier F: Diffuse intrinsic pontine glioma in children: document or treat? World Neurosurg 93:485. e11-485.e14, 2016

11. Puget S, Beccaria K, Blauwblomme T, Roujeau T, James S, Grill J, et al: Biopsy in a series of 130 pediatric diffuse intrinsic pontine gliomas. Childs Nerv Syst 31:1773-1780, 2015

12. Puget $\mathrm{S}$, Blauwblomme T, Grill J: Is biopsy safe in children with newly diagnosed diffuse intrinsic pontine glioma? Am Soc Clin Oncol Educ Book 32:629-633, 2012

13. Robison NJ, Kieran MW: Diffuse intrinsic pontine glioma: a reassessment. J Neurooncol 119:7-15, 2014

14. Roujeau T, Machado G, Garnett MR, Miquel C, Puget S, Geoerger B, et al: Stereotactic biopsy of diffuse pontine lesions in children. J Neurosurg 107 (1 Suppl):1-4, 2007

15. Taylor KR, Mackay A, Truffaux N, Butterfield Y, Morozova O, Philippe C, et al: Recurrent activating ACVR1 mutations in diffuse intrinsic pontine glioma. Nat Genet 46:457-461, 2014

16. Taylor KR, Vinci M, Bullock AN, Jones C: ACVR1 mutations in DIPG: lessons learned from FOP. Cancer Res 74:45654570, 2014

17. Wagner S, Benesch M, Berthold F, Gnekow AK, Rutkowski $S$, Sträter R, et al: Secondary dissemination in children with high-grade malignant gliomas and diffuse intrinsic pontine gliomas. Br J Cancer 95:991-997, 2006

18. Warren KE: Diffuse intrinsic pontine glioma: poised for progress. Front Oncol 2:205, 2012 
19. Wu G, Diaz AK, Paugh BS, Rankin SL, Ju B, Li Y, et al: The genomic landscape of diffuse intrinsic pontine glioma and pediatric non-brainstem high-grade glioma. Nat Genet 46:444-450, 2014

\section{Disclosures}

The authors report no conflict of interest concerning the materials or methods used in this study or the findings specified in this paper.

\section{Author Contributions}

Conception and design: Morales La Madrid, Lobon-Iglesias, Santa-Maria Lopez, Grill. Acquisition of data: Lobon-Iglesias,
Santa-Maria Lopez, Ramos-Albiac, Gomez-Chiari, Bolle, Goumnerova, Kieran, Grill. Analysis and interpretation of data: Morales La Madrid, Lobon-Iglesias, Santa-Maria Lopez, Grill. Drafting the article: Morales La Madrid, Lobon-Iglesias, Santa-Maria Lopez, Grill. Critically revising the article: all authors. Reviewed submitted version of manuscript: Morales La Madrid, Grill. Study supervision: Morales La Madrid, Grill.

\section{Correspondence}

Andres Morales La Madrid: Hospital Sant Joan de Déu, Barcelona, Spain. amorales@hsjdbcn.org. 